\title{
Condylar fractures: is open or closed reduction best?
}

\author{
In patients with fractures of the mandibular condyle, does open or closed \\ reduction produce the best outcomes?
}

\begin{abstract}
Nussbaum ML, Laskin DM, Best AM.
Closed versus open reduction of mandibular condylar fractures in adults: a meta-analysis. J Oral Maxillofac Surg 2008; 66:1087-1927
\end{abstract}

Data sources Medline was searched and further references were identified from selected papers.

Study selection Studies were included if they simultaneously compared open and closed techniques with at least one of the following outcome measures: maximum postoperative mouth opening, amount of lateral excursion and protrusion, mandibular deviation on mouth opening, facial symmetry, and joint or muscle pain. They were also required to have had at least 6 months follow-up and have been published in English.

Data extraction and synthesis Meta-analysis was carried out using the weighted average method for fixed effects and the weighted average method for random effects.

Results Thirteen studies were included, only one of which was a randomised controlled trial (RCT). Numerous problems were found with the information presented in the various articles. These included lack of patient randomisation, failure to classify the type of condylar fracture, variability within the surgical protocols, and inconsistencies in choice of variables and how they were reported.

Conclusions Because of the great variation in how the various study parameters were reported, it was not possible to perform a reliable metaanalysis. There is a need for better standardisation of data collection in future studies as well as randomisation of the patients treated so that the two approaches can be accurately compared.

\section{Commentary}

The mandibular condyle is a common site of fracture, usually because of trauma. The best way to treat such fractures has been the subject of lengthy debate and discussion between oral and maxillofacial surgeons for many years. Treatment of such fractures can be either by surgical open reduction and fixation or by a much simpler closed method, in which the patient is given rigid intermaxillary fixation or, more commonly, intermaxillary elastic traction. An important potential complication of the open approach is damage to the facial nerve. Other techniques including endoscopic approaches are used in some centres. Condylar fractures commonly occur in conjunction with at least one other fracture elsewhere in the mandible, which usually require open reduction and fixation; this may influence the choice of treatment of the condyle.

The literature is littered with numerous studies that have attempted to address which modality of treatment is best. The problem with most of these is they are non-randomised, often retrospective case series, with mismatched groups and a multitude of outcome measures reported, as highlighted in this review. The authors did identify a RCT by Worsae and Thorn ${ }^{1}$ but with no clear method of randomisation, allocation concealment or blinding (which would be difficult for such a trial) and significant dropouts, this is not a good-quality RCT.

One of the main shortcomings of this review is that it failed to search the literature adequately using a fairly conservative and haphazard search strategy. A more comprehensive search and some clearly defined inclusion and exclusion criteria, as in a Cochrane review, would have improved this, and such a review is presently being undertaken.

The only other credible international multicentre RCT, by Eckelt et al. published in 2006, ${ }^{2}$ was not identified here. The Eckelt study concluded that either modality of treatment of condylar fractures yielded acceptable results but, for the outcomes assessed, the authors reported superior results with the open method and no cases of permanent damage to the facial nerve.

Nussbaum and co-authors highlighted the heterogeneity of published studies but proceeded with a meta-analysis of some outcome measures, which is not helpful. This intervention comparison would be well suited to a so-called pragmatic RCT using more patient-centred outcomes and particularly quality of life. This is because, ultimately, clinicians can undertake all manner of measurements of mandibular function, mouth opening and facial appearance, but what matters to the patients is how they feel undergoing each of these treatments and, in the longer term, how well they function in their everyday living.

\section{Richard Oliver}

School of Dentistry, University of Manchester, Manchester, UK

1. Worsaae N, Thorn JJ. Surgical versus nonsurgical treatment of unilateral dislocated low subcondylar fractures: a clinical study of 52 cases. J Oral Maxillofac Surg 1994; 52:353-360.

2. Eckelt $U$, Schneider M, Erasmus $F$, et al. Open versus closed treatment of fractures of the mandibular condylar process-a prospective randomized multi-centre study. J Craniomaxillofac Surg 2006; 34:306-314.

Evidence-Based Dentistry (2008) 9, 84. doi:10.1038/sj.ebd.6400601 